tively these articles will give readers a clear sense of how far we have come, what essential questions remain to be answered, and how a rigorous, collaborative scientific community can help to answer them. Today more than ever, the importance of independent scientific advisors and agencies is clear.

Disclosure forms provided by the authors are available with the full text of this editorial at NEJM.org.

From the National Academy of Medicine, Washington, DC (V.J.D.); the Gordon and Betty Moore Foundation, Palo Alto, CA
(H.V.F.); the University of Texas System, Austin (K.I.S.); and Harvard Medical School, Boston (S.O.T.). Dr. Dzau is the current president of the National Academy of Medicine; Drs. Fineberg, Shine, and Thier are past presidents of the Institute of Medicine/National Academy of Medicine.

1. Institute of Medicine. To improve human health: a history of the Institute of Medicine. Washington, DC: National Academies Press, 1998 (https://www.nap.edu/read/6382/chapter/1).

2. Fauci AS, Lane HC. Four decades of HIV/AIDS - much accomplished, much to do. N Engl J Med 2020;383:1-4.

DOI: 10.1056/NEJMe2020118

Copyright (c) 2020 Massachusetts Medical Society.

\title{
Antibiotics for Childhood Pneumonia - Do We Really Know How Long to Treat?
}

\author{
Anne B. Chang, F.R.A.C.P., Ph.D., and Keith Grimwood, F.R.A.C.P., M.D.
}

The World Health Organization (WHO) recommends 3 days of oral amoxicillin $(80 \mathrm{mg}$ per kilogram of body weight per day) in children younger than 5 years of age who have fast-breathing pneumonia (cough lasting $<14$ days or difficulty breathing, along with fast breathing for age) and are living in areas with a low prevalence of human immunodeficiency virus. ${ }^{1}$ If retractions of the chest wall are present, 5 days of amoxicillin are recommended. In children with severe clinical pneumonia and danger signs (e.g., dehydration or reduced level of consciousness), 5 days of intravenous antibiotic agents are recommended. In this issue of the Journal, two well-performed randomized, controlled trials challenge these recommendations in young children with nonsevere pneumonia: a trial by Jehan et al. conducted in Pakistan (RETAPP, Randomized Trial of Amoxicillin versus Placebo for [Fast Breathing] Pneumonia) ${ }^{2}$ and a trial by Ginsburg et al. conducted in Malawi. ${ }^{3}$ Both trials had a noninferiority design and were performed in lowincome communities in which pneumonia remains the leading cause of death in children younger than 5 years of age.

RETAPP included 4002 children with pneumonia with tachypnea with or without wheezing and without retraction of the chest wall (if wheeze was present, children received up to three doses of an inhaled bronchodilator). The trial by Ginsburg et al. enrolled 3000 children with pneumo- nia with retractions (i.e., chest-indrawing pneumonia, defined as cough lasting < 14 days or difficulty breathing, along with visible indrawing of the chest wall with or without fast breathing for age); wheezing status was not reported. In both trials, three levels (or "bands") of amoxicillin dosing were used (dosing was determined on the basis of weight in RETAPP and on the basis of age in the trial by Ginsburg et al.); both trials used a lower limit of 50 to $75 \mathrm{mg}$ per kilogram per day. Jehan et al. found that by day 3, the incidence of treatment failure (the primary outcome) was higher in the placebo group (4.9\%) than in the amoxicillin group (2.6\%) - a difference that was above the noninferiority margin of 1.75 percentage points. At day 14, relapse had occurred in 40 children $(2.2 \%)$ in the placebo group and in 58 children (3.1\%) in the amoxicillin group (mean difference, 0.9 percentage points; $95 \%$ confidence interval [CI], -2.1 to 0.3 ). In the trial by Ginsburg et al., the incidence of treatment failure at day 6 was 5.9\% among children who received 3 days of amoxicillin and 5.2\% among children who received 5 days of amoxicillin - a result that met the criterion for noninferiority (an incidence of treatment failure in the 3-day group that was no more than 1.5 times that in the 5-day group). By day 14, the absolute between-group difference in the percentage of children who had either treatment failure before or on day 6 or relapse by day 14 was 1.7 percent- 
age points ( $95 \% \mathrm{CI},-0.7$ to 4.1 ), favoring the 5-day group.

These two trials are important, but their results are insufficient to change current clinical practice. The results of RETAPP support current WHO guidelines, whereas the findings in the trial by Ginsburg et al. indicate that 3 days of amoxicillin is sufficient for treatment of chestindrawing pneumonia. However, the relative noninferiority margin in that trial of a $50 \%$ higher incidence of treatment failure in the 3-day group than in the 5-day group is large. Additional data from randomized, controlled trials or from metaanalyses that use individual patient data from several large randomized, controlled trials with smaller relative margins conducted in various settings would be required for the current results to change practice. Furthermore, the results of these two trials cannot be extrapolated to other definitions of pneumonia. There is little information to guide the appropriate duration of treatment in patients with pneumonia confirmed on chest radiography. The results of a singlecenter, three-group, randomized, controlled trial in Israel involving 140 nonhospitalized children younger than 5 years of age with probable bacterial pneumonia (determined on the basis of clinical criteria, chest radiography, and white-cell counts) showed a high incidence of treatment failure (40\%) among children who received a 3 -day course of amoxicillin ( $80 \mathrm{mg}$ per kilogram per day); this finding resulted in the 3-day regimen being abandoned in that trial. ${ }^{4}$ In addition, in contrast to WHO guidelines, current national guidelines in high-income countries recommend antibiotics for 5 to 10 days. $^{5}$

The two current trials highlight our limited knowledge regarding appropriate antibiotic prescribing for the various types of early childhood pneumonia as categorized according to $\mathrm{WHO}$ criteria. ${ }^{1}$ Both trials followed current WHO treatment guidelines, which sacrifice specificity for sensitivity to reduce mortality in low-income countries. Nevertheless, in RETAPP, the number of deaths was very low, even in the placebo group ( 1 of 1999 children [0.05\%] died), possibly because most participants had viral infections. Indeed, the criteria for administration of antibiotics need to be assessed further in low-income areas, especially considering that in RETAPP the number needed to treat to prevent one treatment failure was 44. In most clinical settings, fever is one of the key symptoms in childhood pneumonia. Yet in both trials, the recorded incidences of fever and hypoxia were low: in RETAPP, $32.6 \%$ of participants had a body temperature of at least $37.5^{\circ} \mathrm{C}$, and $12.7 \%$ had an oxygen saturation of less than $93 \%$. In the trial by Ginsburg et al., 30.9\% of participants had a body temperature of at least $38.0^{\circ} \mathrm{C}$, and $0.4 \%$ had an oxygen saturation of less than $93 \%$. Not surprisingly, RETAPP showed that respiratory rate and fever independently predicted treatment failure. An unexpected finding was that wheezing (present in approximately $7 \%$ of participants) was an independent predictor of treatment failure (odds ratio, 1.89; $95 \%$ CI, 1.14 to 3.14) - a result that indicates that amoxicillin may be beneficial even when wheezing (which is usually assumed to signify nonbacterial pneumonia ${ }^{6}$ ) is present. Because of the low-income settings in which the trials were conducted, the fact that neither trial included data on chest radiography or microbiologic data is understandable. Clinical criteria alone are clearly insufficient to diagnose bacterial pneumonia, and biomarkers and other objective measures are needed.

The findings in these two fairly short-term trials are emblematic of the persistently high prevalence of malnutrition and the suboptimal prevalence of immunization (approximately 50 to $60 \%$ in these countries. Research on preventing pneumonia and studies of longer-term outcomes are still required. Data from studies that examine the duration of chronic symptoms (e.g., wet cough lasting $>4$ weeks), respiratory-associated hospitalizations, or persistent abnormalities on chest radiography after pneumonia treatment are lacking. Whether longer courses of antibiotics may benefit some children with pneumonia is unknown, but such benefit is plausible because the longer course would increase the likelihood of pathogen clearance from the airways and reduce the risk of persistent inflammation and airway injury. Indeed, the results of trials involving children younger than 2 years of age with otitis media ${ }^{7}$ have shown poorer clinical outcomes with shorter courses of antibiotics. Prospective data have shown that pneumonia may reduce future lung function ${ }^{8}$ and shape lungfunction trajectories'; thus, pneumonia and its consequences have public health implications. A 
prospective study involving 126,359 adults 35 to 70 years of age from 17 countries who were followed for a median of 7.8 years (interquartile range, 5.6 to 9.5) showed that lung-function impairment had a greater effect on the risk of future cardiorespiratory illness and death than tobacco smoking. ${ }^{10}$ These important trials conducted by Jehan et al. and Ginsburg et al. have contributed to our current knowledge, yet many gaps remain in our understanding of the appropriate management of pneumonia and deserve greater attention.

Disclosure forms provided by the authors are available with the full text of this editorial at NEJM.org.

From the Australian Centre for Health Services Innovation, Queensland University of Technology, and the Department of Respiratory and Sleep Medicine, Queensland Children's Hospital, Brisbane (A.B.C.), the Menzies School of Health Research, Darwin, QLD (A.B.C.), and the School of Medicine and Menzies Health Institute Queensland, Griffith University, and the Departments of Infectious Diseases and Paediatrics, Gold Coast Health, Gold Coast (K.G.) — all in Australia.

1. Revised WHO classification and treatment of childhood pneumonia at health facilities. Geneva: World Health Organization, 2014.

2. Jehan F, Nisar I, Kerai S, et al. Randomized trial of amoxi- cillin for pneumonia in Pakistan. N Engl J Med 2020;383:2434.

3. Ginsburg A-S, Mvalo T, Nkwopara E, et al. Amoxicillin for 3 or 5 days for chest-indrawing pneumonia in Malawian children. N Engl J Med 2020;383:13-23.

4. Greenberg D, Givon-Lavi N, Sadaka Y, Ben-Shimol S, Bar-Ziv J, Dagan R. Short-course antibiotic treatment for communityacquired alveolar pneumonia in ambulatory children: a doubleblind, randomized, placebo-controlled trial. Pediatr Infect Dis J 2014;33:136-42.

5. Katz SE, Williams DJ. Pediatric community-acquired pneumonia in the United States: changing epidemiology, diagnostic and therapeutic challenges, and areas for future research. Infect Dis Clin North Am 2018;32:47-63.

6. Duke T. What the PERCH study means for future pneumonia strategies. Lancet 2019;394:714-6.

7. Hoberman A, Paradise JL, Rockette HE, et al. Shortened antimicrobial treatment for acute otitis media in young children. N Engl J Med 2016;375:2446-56.

8. Gray DM, Turkovic L, Willemse L, et al. Lung function in African infants in the Drakenstein child health study: impact of lower respiratory tract illness. Am J Respir Crit Care Med 2017; 195:212-20.

9. Bui DS, Lodge CJ, Burgess JA, et al. Childhood predictors of lung function trajectories and future COPD risk: a prospective cohort study from the first to the sixth decade of life. Lancet Respir Med 2018;6:535-44.

10. Duong M, Islam S, Rangarajan S, et al. Mortality and cardiovascular and respiratory morbidity in individuals with impaired FEV $_{1}$ (PURE): an international, community-based cohort study. Lancet Glob Health 2019;7(5):e613-e623.

DOI: 10.1056/NEJMe2016328

Copyright (C) 2020 Massachusetts Medical Society. 